September 2005

SLAC-PUB-11459

\title{
Spectroscopy and New Particles from BABAR
}

\author{
B. Aa. Petersen ${ }^{\text {a }}$ \\ a Stanford University Physics Department, \\ 382 Via Pueblo Mall, Stanford, CA 94305-4060, USA \\ Results on charmonium states and searches for pentaquark states at the BABAR experiment are presented. \\ Submitted to Nuclear Physics B - Proceedings Supplements
}

\section{INTRODUCTION}

The field of hadron spectroscopy has undergone a revival in recent years with the observation of many new states. Some of these states, such as $D_{s J}(2317)^{+}$and $D_{s J}(2460)^{+}$, are now well established and appear to be ordinary charm mesons. Others, such as $X(3872)$ and $Y(3940)$, could be new excited charmonium $(c \bar{c})$ states, but require more measurements for a definite identification. Observation of the pentaquark state $\Theta_{5}(1540)^{+}$ has been reported by about a dozen experiments, while many other experiments see no evidence for this state and in fact some are in direct contradiction of some of the observations.

The BABAR experiment [1] is an $e^{+} e^{-}$collider experiment running at or just below the $\Upsilon(4 S)$ resonance. It was designed to perform precision measurements of $\mathrm{CP}$ violation in the $B$ system, but has proven to have a significantly broader physics reach. Among many things, it was the first experiment to observe the $D_{s J}(2317)^{+}$ state [2]. Here we report on recent results on charmonium(-like) states and the search for pentaquarks using the BABAR data sample.

\section{STUDIES OF THE $X(3872)$ STATE}

The $X(3872)$ state was first observed [3] by Belle in studies of $B^{ \pm} \rightarrow K^{ \pm} J / \psi \pi^{+} \pi^{-}$decays. In the invariant mass spectrum of the $J / \psi \pi^{+} \pi^{-}$system $\left(m\left(J / \psi \pi^{+} \pi^{-}\right)\right)$, a narrow signal is evident at about $3872 \mathrm{MeV} / \mathrm{c}^{2}$. This could be another charmonium state, but the measured mass does not seem compatible with existing potential models. Its mass is also very close to the threshold for
$D^{0} \bar{D}^{* 0}$ production. For these reasons, other explanations have been proposed; for example, it could be a weakly bound molecule-like state of $D \bar{D}^{*}$ or a tetraquark state. To try to discriminate among the different models, $B A B A R$ has studied the $X(3872)$ state in several ways.

Using 232 million $B \bar{B}$ pairs recorded by $B A B A R$, $X(3872)$ candidates are reconstructed [4] in the decay modes $B^{ \pm} \rightarrow K^{ \pm} J / \psi \pi^{+} \pi^{-}$and $B^{0} \rightarrow$ $K_{S}^{0} J / \psi \pi^{+} \pi^{-}$, where $J / \psi$ decays into di-leptons and the $K_{S}^{0}$ into oppositely-charged pions. $B$ decays are selected using two kinematic variables: $\Delta E=E_{B}^{*}-\sqrt{s} / 2$ and the energy-substituted mass $m_{\mathrm{ES}}=\sqrt{\left(s / 2+\mathbf{p}_{0} \cdot \mathbf{p}_{B}\right)^{2} / E_{0}^{2}-\mathbf{p}_{B}^{2}}$. Here $E_{B}^{*}$ is the energy of the $B$ meson candidate in the center-of-mass $(\mathrm{CM})$ frame, $E_{0}$ and $\sqrt{s}$ are the total energies of the $e^{+} e^{-}$system in laboratory and $\mathrm{CM}$ frames, respectively; $\mathbf{p}_{0}$ and $\mathbf{p}_{B}$ are the three-momenta of the $e^{+} e^{-}$system and the $B$ candidate in the laboratory frame, respectively.

Fig. 1 shows the $m\left(J / \psi \pi^{+} \pi^{-}\right)$distributions for the two $B$ decay modes. In each case the signal yield of $B \rightarrow X(3872) K$ decays is extracted using an unbinned maximum likelihood fit to $m_{\mathrm{ES}}$ and $m\left(J / \psi \pi^{+} \pi^{-}\right)$. For the $B^{ \pm}$decay mode we obtain $61 \pm 15$ events, while for the $B^{0}$ decay mode, only $8.3 \pm 4.5$ events. Including systematic uncertainties, the signals are found to have a significance of $6.1 \sigma$ and $2.5 \sigma$, respectively. The signal yields correspond to the branching fractions $\mathcal{B}^{-}=\mathcal{B}\left(B^{-} \rightarrow X(3872) K^{-}, X \rightarrow J / \psi \pi^{+} \pi^{-}\right)=$ $(10.1 \pm 2.5$ (stat. $) \pm 1.0$ (syst. $)) \times 10^{-6}$ and $\mathcal{B}^{0}=$ $\mathcal{B}\left(B^{0} \rightarrow X(3872) K^{0}, X \rightarrow J / \psi \pi^{+} \pi^{-}\right)=(5.1 \pm$ 2.8 (stat. $) \pm 0.7$ (syst. $)) \times 10^{-6}$. From these we obtain a ratio of branching fractions, $R=\mathcal{B}^{0} / \mathcal{B}^{-}=$ 

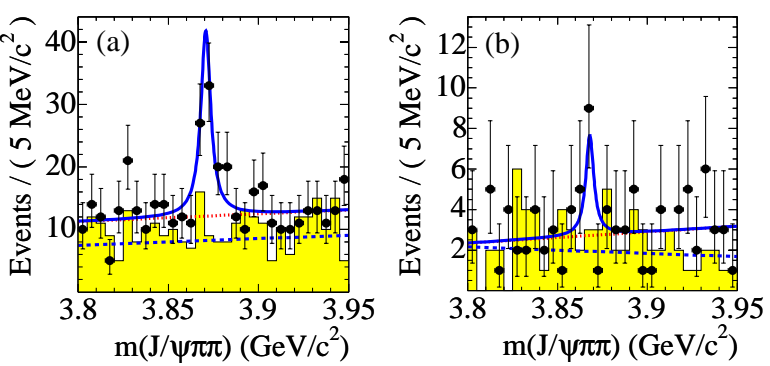

Figure 1. Signal region projections of $m\left(J / \psi \pi^{+} \pi^{-}\right)$for (a) $B^{-} \rightarrow X(3872) K^{-}$and (b) $B^{0} \rightarrow X(3872) K_{S}^{0}$. The dashed and dotted curves show the estimated combinatorial and non-resonant background. The shaded region shows events in an $m_{\mathrm{ES}}$ sideband region.

$0.50 \pm 0.30$ (stat. $) \pm 0.05$ (syst.).

The invariant mass distribution of the $\pi^{+} \pi^{-}$in $X(3872) \rightarrow J / \psi \pi^{+} \pi^{-}$decays, suggests that the decay may proceed through a $\rho^{0}$ resonance. If so, one can expect to find charged isospin partners, $X(3872)^{ \pm}$, of the $X(3872)$. We have searched [5] for these in the decays $B^{-} \rightarrow K_{S}^{0} J / \psi \pi^{-} \pi^{0}$ and $B^{0} \rightarrow K^{+} J / \psi \pi^{-} \pi^{0}$ using the same technique as for the neutral $X(3872)$. No evidence of a charged $X(3872)$ is found and we set the following limits at $90 \%$ confidence level (CL): $\mathcal{B}\left(B^{-} \rightarrow X^{-} K_{S}^{0}, X^{-} \rightarrow J / \psi \pi^{-} \pi^{0}\right)<11 \times 10^{-6}$ and $\mathcal{B}\left(B^{0} \rightarrow X^{-} K^{+}, X^{-} \rightarrow J / \psi \pi^{-} \pi^{0}\right)<5.4 \times$ $10^{-6}$.

\section{INCLUSIVE $B^{ \pm} \rightarrow X_{c \bar{c}} K^{ \pm}$RECON- STRUCTION}

An alternative method of observing charmonium states in $B$ decays involves reconstructing everything except the charmonium state itself. Charged $B$ mesons are fully reconstructed [6] in many different decay modes, $D^{(*)} n H$, where $H$ is a combination of $\pi^{ \pm}, \pi^{0}, K^{ \pm}$and $K_{S}^{0}$ hadrons. In $210 \mathrm{fb}^{-1}$ of data, about $380,000 B^{ \pm}$mesons are fully reconstructed. The fully-reconstructed $B^{ \pm}$meson defines the rest frame of the second $B$ meson. A neural network is used to select a

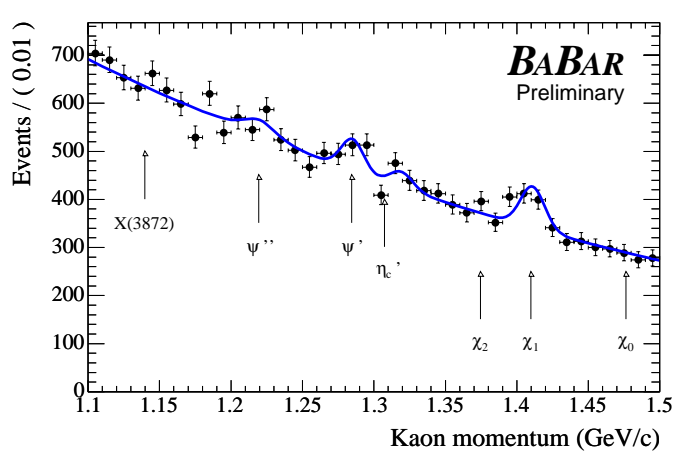

Figure 2. Kaon momentum in the $B$ meson rest frame. The arrows show the expected positions of the charmonium states.

Table 1

Absolute branching fractions for $B^{ \pm} \rightarrow X_{c \bar{c}} K^{ \pm}$. Upper limits are given at 90\% CL.

\begin{tabular}{|c|c|c|c|}
\hline Particle & $\mathcal{B}\left(10^{-4}\right)$ & Particle & $\mathcal{B}\left(10^{-4}\right)$ \\
\hline$\eta_{c}$ & $8.9 \pm 1.5$ & $\overline{\eta_{c}^{\prime}}$ & $3.1 \pm 1.5$ \\
\hline$J / \psi$ & $8.1 \pm 1.6$ & $\psi^{\prime}$ & $4.2 \pm 1.4$ \\
\hline$\chi_{c 0}$ & $<1.8$ & $\psi^{\prime \prime}$ & $3.2 \pm 2.3$ \\
\hline $\begin{array}{l}\chi_{c 1} / h_{c} \\
\chi_{c 2}\end{array}$ & $\begin{array}{l}7.0 \pm 1.6 \\
<2.0\end{array}$ & $X(3872)$ & $<3.2$ \\
\hline
\end{tabular}

kaon from the decay of the second $B$ meson. The kaon momentum in the $B$ rest frame is related to the mass $\left(m_{X}\right)$ of the system recoiling against it by $m_{X}=\sqrt{m_{B}^{2}+m_{K}^{2}-2 E_{K} m_{B}}$. Any twobody decay $B^{ \pm} \rightarrow X_{c \bar{c}} K^{ \pm}$should yield a signal in the kaon momentum spectrum at the momentum corresponding to the $X_{c \bar{c}}$ value.

The kaon momentum spectrum shows clear signals for $J / \psi$ and $\eta_{c}$ mesons, and, as shown in Fig. 2, several excited charmonium signals are also present. An unbinned maximum likelihood fit is used to extract the yields for nine known charmonium states. Together with the number of reconstructed $B^{ \pm}$mesons and estimated efficiencies, this allows us to measure or set limits on the absolute branching fractions of all nine modes. The results are listed in Table 1. 


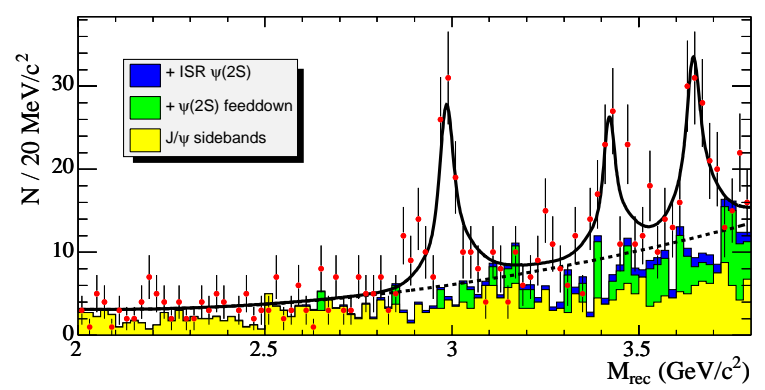

Figure 3. Inclusive mass recoiling against a $J / \psi$. The dashed curve represents the background component from the fit. The histograms indicate different sources of background.

From the upper limit on $\mathcal{B}\left(B^{ \pm} \rightarrow\right.$ $\left.X(3872) K^{ \pm}\right)$and the measurement of $\mathcal{B}\left(B^{-} \rightarrow\right.$ $\left.X(3872) K^{-}, X \rightarrow J / \psi \pi^{+} \pi^{-}\right)$, we estimate $\mathcal{B}\left(X(3872) \rightarrow J / \psi \pi^{+} \pi^{-}\right)>4.3 \%$ at $90 \%$ CL.

\section{DOUBLE CHARMONIUM PRODUC- TION}

In $B A B A R$ charmonium states are produced not only in $B$ decays. We have measured [7] the cross sections for double charmonium production in the process $e^{+} e^{-} \rightarrow \gamma^{*} \rightarrow J / \psi c \bar{c}$ using $124 \mathrm{fb}^{-1}$ of data. Only $c \bar{c}$ states with even C-parity are expected, although if there is a contribution from $e^{+} e^{-} \rightarrow \gamma^{*} \gamma^{*} \rightarrow J / \psi c \bar{c}$, odd C-parity states can also be produced.

The $J / \psi$ mesons are reconstructed in their dilepton decay modes and used to calculate the mass of the recoil system. The main background is from $J / \psi$ mesons produced in ISR and other QED processes. This is suppressed by requiring at least five charged tracks in each event and limiting the corresponding missing momentum.

The recoil mass spectrum is shown in Fig. 3. Three even C-parity charmonium states, $\eta_{c}, \chi_{c 0}$ and $\eta_{c}^{\prime}$, are observed, while there is no evidence for odd C-parity states. The distribution is fit to obtain the yield for each state, from which the production cross section is calculated. Due to the requirement of at least five

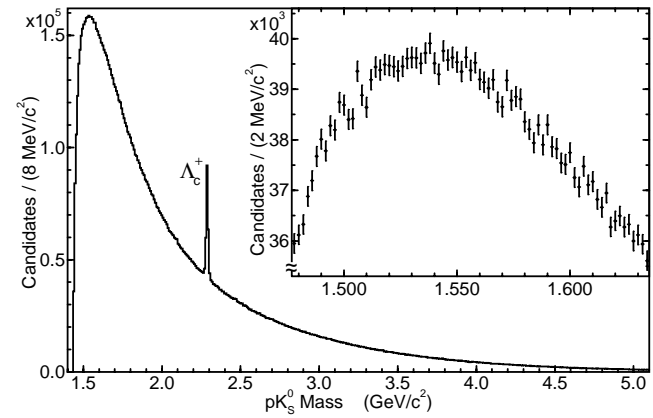

Figure 4. Distribution of the $p K_{S}^{0}$ invariant mass. The inset shows the region where a $\Theta_{5}(1540)^{+}$ state should manifest itself.

tracks in the event, we report the product of the branching fraction to states with more than two charged tracks and the production cross section, $\mathcal{B}(c \bar{c} \rightarrow>2$ charged $) \sigma\left(e^{+} e^{-} \rightarrow J / \psi c \bar{c}\right)$. The results are $17.6 \pm 2.8_{-2.1}^{+1.5} \mathrm{fb}, 10.3 \pm 2.5_{-1.8}^{+1.4} \mathrm{fb}$ and $16.4 \pm 3.7_{-3.0}^{+2.4} \mathrm{fb}$ for $J / \psi \eta_{c}, J / \psi \chi_{c 0}$ and $J / \psi \eta_{c}^{\prime}$, respectively. These cross sections are much larger than those predicted by NRQCD calculations [8].

\section{PENTAQUARK SEARCHES}

After the first report [9] of the pentaquark $\Theta_{5}(1540)^{+}$, many other experiments have reported evidence of the same state, while one experiment [10] has reported evidence for the $\Xi_{5}(1860)^{--}$pentaquark state. BABAR has searched for both of these states in several different production scenarios.

\subsection{Inclusive $e^{+} e^{-}$production}

Using a data sample of $123 \mathrm{fb}^{-1}$ we have performed a search [11] for inclusive production in $e^{+} e^{-}$annihilation of the two pentaquark states. The states are sought in the decay modes $\Theta_{5}^{+} \rightarrow$ $p K_{s}^{0}$ and $\Xi_{5}^{--} \rightarrow \Xi^{-} \pi^{-}$and the corresponding decay vertices are required to come from $e^{+} e^{-}$ collision region. The secondary decays are reconstructed in the modes $K_{S}^{0} \rightarrow \pi^{+} \pi^{-}$and $\Xi^{-} \rightarrow$ $\Lambda \pi^{-}, \Lambda \rightarrow p \pi^{-}$.

Fig. 4 shows the invariant mass spectrum of all selected $p K_{S}^{0}$ candidates. A clear signal for 
$\Lambda_{c}^{+} \rightarrow p K_{S}^{0}$ is seen, but there is no evidence of any $\Theta_{5}^{+}$state. The mass resolution in the $\Theta_{5}^{+}$region is about $2 \mathrm{MeV} / c^{2}$. Similarly the invariant mass spectrum for the $\Xi^{-} \pi^{-}$candidates shows no evidence of a $\Xi_{5}^{--}$state. We set upper limits at $95 \%$ CL on the total production cross section for these two states in $e^{+} e^{-}$annihilations of $171 \mathrm{fb}$ and $25 \mathrm{fb}$ for $\Theta_{5}^{+}$and $\Xi_{5}^{--}$, respectively. These limits assume widths of $1 \mathrm{MeV} / c^{2}, \mathcal{B}\left(\Theta_{5}^{+} \rightarrow\right.$ $\left.p K_{S}^{0}\right)=0.25$ and $\mathcal{B}\left(\Xi_{5}^{--} \rightarrow \Xi^{-} \pi^{-}\right)=0.5$. The limits are about eight and four times lower than the rates measured in $e^{+} e^{-}$collisions for ordinary baryons of similar mass.

\subsection{Electro- and hadro-production}

A second search for $\Theta_{5}^{+} \rightarrow p K_{S}^{0}$ has been performed by studying $p K_{S}^{0}$ candidates inconsistent with coming from the $e^{+} e^{-}$collision region. These particles are produced in interactions with detector material. The incoming particles are hadrons from a primary interaction (hadro-production), off-momentum beam particles (electro-production) or other background particles. The distribution of the $p K_{s}^{0}$ vertices is consistent with the material distribution in $B A B A R$. The invariant mass distribution of the $p K_{S}^{0}$ candidates, shown in Fig. 5, shows no evidence of a $\Theta_{5}^{+}$pentaquark. Restricting the data sample to candidates in which a third non-baryon particle is coming from the same vertex also shows no evidence for $\Theta_{5}^{+}$. About $25 \%$ of the candidates in Fig. 5 come from beam particles interacting in a small region of the detector made primarily of Beryllium. Selecting just events from that particular region allows us to study events from electro-production in Beryllium, but again no signal for $\Theta_{5}^{+}$is seen.

\section{SUMMARY}

The BABAR experiment is in an excellent position to study charmonium states. This paper summarizes several of the techniques used at $B A B A R$ to measure production cross sections and branching fractions for a variety of such states and to search for new states.

We have also searched for $\Theta_{5}(1540)^{+}$and $\Xi_{5}(1860)^{--}$in inclusive $e^{+} e^{-}$production and

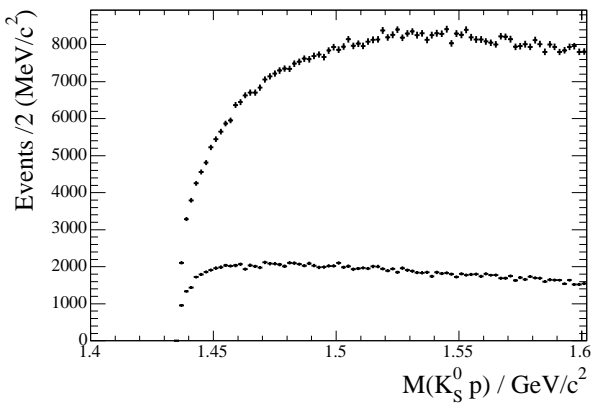

Figure 5. Invariant mass distribution of $p K_{S}^{0}$ candidates produced in material interaction. The upper points are from the $K_{S}^{0}$ signal region, while the lower points are from $K_{S}^{0}$ mass sidebands.

for $\Theta_{5}(1540)^{+}$production in particle interactions with detector material. No evidence of pentaquark production has been found at BABAR.

\section{REFERENCES}

1. B. Aubert et al. (BABAR Collaboration), Nucl. Instr. Methods Phys. Res., Sect. A 479, 1 (2002).

2. B. Aubert et al. (BABAR Collaboration), Phys. Rev. Lett. 90, 242001 (2003).

3. S.-K. Choi et al. (Belle Collaboration), Phys. Rev. Lett. 91, 262001 (2003).

4. B. Aubert et al. (BABAR Collaboration), Submitted to Phys. Rev. Lett., hep-ex/0507090.

5. B. Aubert et al. (BABAR Collaboration), Phys. Rev. D 71, 031501 (2005).

6. B. Aubert et al. (BABAR Collaboration), Phys. Rev. Lett. 92, 071802 (2004).

7. B. Aubert et al. (BABAR Collaboration), Phys. Rev. D 72, 031101 (2005).

8. E. Braaten and V. L. Chernyak, Phys. Lett. B 612, 215 (2005).

9. T. Nakano et al. (LEPS Collaboration), Phys. Rev. Lett. 91, 012002 (2003).

10. C. Alt et al. (NA49 Collaboration), Phys. Rev. Lett. 92, 042003 (2004).

11. B. Aubert et al. (BABAR Collaboration), Phys. Rev. Lett. 95, 042002 (2005). 